\title{
THE STABILITY OF A MULTIPLE ALLELIC SYSTEM
}

\author{
S. P. H. MANDEL \\ Department of Genetics, Combridge
}

\section{INTRODUCTION}

Received I.viii. $5^{8}$

A multiple allelic system at a locus can give rise to genotypes of different viabilities. Under certain conditions the genes comprising such a system can exist in equilibrium in a large random mating population, giving rise to the situation known as a (balanced) polymorphism. Owen (1953, I954), in discussing the problem for three alleles, has stated a set of necessary and sufficient conditions for such an equilibrium to be stable with respect to small variations of the gene frequencies about their equilibrium values. The object of this paper is to present formal proofs of these conditions for the general case of $n$ alleles, and to investigate, in some detail, the three-allele situation. In order to do this the question of the change in mean viability of the population is studied in the manner indicated by Mandel and Hughes (r958).

The situation to be investigated is that in which there are discrete, non-overlapping generations. Kimura (1956) and Penrose et al. (1956) have studied the problem, introducing a continuous time parameter, and replacing recurrence relations by differential equations. This procedure assumes that the change in the state of the population from one generation to the next is continuous, and is justified only in the case of infinitesimally slow selection. The analysis to be given here is free from any such restriction.

\section{CONDITIONS FOR A NON-TRIVIAL EQUILIBRIUM}

In general, consider $n$ alleles $A_{1}, A_{2}, \ldots, A_{n}$ at a single locus, in a large population mating at random. Let $a_{i j}$ denote the relative viability of the genotype $A_{i} A_{j}(i, j=\mathrm{r}, 2, \ldots, n)$; the $a_{i j}$ thus form a symmetric matrix $\left(a_{i j}\right)$ and we write

$$
\Delta=\operatorname{det}\left(a_{i j}\right) \text {. }
$$

Let $p_{i}(i=\mathrm{I}, 2, \ldots, n)$ denote the frequency of gene $A_{i}(i=\mathrm{I}, 2, \ldots, n)$ in the population in any one generation, so that the $p_{i}$ describe the state of the population and

Let

$$
\sum_{i=\mathrm{x}}^{n} p_{i}=\mathrm{I}, \quad 0 \leqslant p_{i} \leqslant \mathrm{I} .
$$

$$
V=\sum_{\substack{i=\mathrm{x} \\ j=\mathrm{x} \\ 289}}^{n} \sum_{i j}^{n} p_{i} p_{j}
$$


$V$ is thus a function of the gene frequencies, and will be called the mean viability of the population. If the population in state $p_{i}$ undergoes random mating, the frequencies $p_{i}^{\prime}$ in the following generation will be given by the relations

$$
V p_{i}^{\prime}=p_{i} \sum_{j=1}^{n} a_{i j} p_{j} \quad(\text { all } i=\mathrm{I}, 2, \ldots, n) .
$$

The population is in equilibrium if, for all $i, p_{i}^{\prime}=p_{i}$. This can happen in a variety of ways in which one or more of the $p_{i}$ vanish. The non-trivial equilibrium, in which none of the $p_{i}$ is zero, is defined (if it exists) by the relations

$$
\sum_{j=1}^{n} a_{i j} p_{j}=V, \text { for all } i=\mathrm{I}, 2, \ldots, n .
$$

Let $P_{i}(i=\mathrm{I}, 2, \ldots, n)$ denote the values of the $p_{i}$ satisfying this set of inhomogeneous equations, and $V_{e}$ the corresponding value of $V$, then

$$
P_{i}=\frac{\sum_{i=1}^{n} A_{i j}}{\sum_{i=\mathrm{x}}^{n} \sum_{j=\mathrm{x}}^{n} A_{i j}}=\frac{\sum_{j=\mathrm{x}}^{n} A_{i j}}{D}
$$

where $A_{i j}$ denotes the cofactor of $a_{i j}$ in $\Delta$, and writing

Also

$$
D=\sum_{i=1}^{n} \sum_{j=1}^{n} A_{i j} \text {. }
$$

$$
V_{e}=\frac{\Delta}{D}
$$

and setting

(6) becomes

$$
A_{i}=\sum_{j=\mathbf{x}}^{n} A_{i j}, \quad \text { all } i=\mathrm{I}, 2, \ldots, n
$$

$$
\left.\begin{array}{l}
P_{i}=\frac{A_{i}}{D}, \text { all } i=1,2, \ldots, n \\
P_{i}=\frac{V_{e} A_{i}}{\Delta}, \quad \text { all } i=1,2, \ldots, n .
\end{array}\right\}
$$

In ordcr that an equilibrium may exist, it is necessary that the $P_{i}$ all be positive; this means that the $A_{i}$ must all have the same sign, and this will also be the sign of $D$ and of $\Delta$. If this condition does not hold, no non-trivial cquilibrium is possible. Moreover, it follows from (5) that there can be at most one non-trivial state of equilibrium. 


\section{THE CHANGE IN MEAN VIABILITY IN SUCCESSIVE GENERATIONS}

In this section the variation of $V$, the mean viability, from one generation to the next will be examined when the population is in the neighbourhood of an (existent) equilibrium state; it will be shown that the value of $V$ increases each time the population undergoes random mating.

Suppose, therefore, that a polymorphic equilibrium exists, i.e. the $A_{i}$ have the same sign for all $i=\mathrm{I}, 2, \ldots, n$.

Let $P_{i}(i=\mathrm{I}, 2, \ldots, n)$ denote the equilibrium gene frequencies as before and $V_{e}$ the corresponding value of $V$.

Consider the population in the state $p_{i}(i=1,2, \ldots, n)$ near the equilibrium, so that

$$
p_{i}=P_{i}+x_{i}, \quad(i==\mathrm{I}, 2, \ldots, n)
$$

where the $x_{i}$ are small and

Also write

$$
\sum_{i=1}^{n} x_{i}=0
$$

and

$$
X_{i}=\sum_{j=x}^{n} a_{i j} x_{j}, \quad(i=\mathrm{I}, 2, \ldots, n)
$$

In the next generation

$$
\delta=\sum_{i=1}^{n} x_{i} X_{i}=\sum_{i=\mathrm{x}}^{n} \sum_{j=\mathrm{x}}^{n} a_{i j} x_{i} x_{j} .
$$

Also

$$
\begin{aligned}
V p_{i}^{\prime} & =p_{i} \sum_{j=1}^{n} a_{i j} p_{j} \\
& =p_{i}\left(V_{e}+X_{i}\right)
\end{aligned}
$$

$$
\begin{aligned}
V & =\sum_{i=1}^{n} p_{i} \sum_{j=x}^{n} a_{i j} p_{j} \\
& =\sum_{i=1}^{n} p_{i}\left(V_{e}+X_{i}\right) \\
& =V_{e}+\sum_{i=1}^{n} x_{i} X_{i}+\sum_{i=1}^{n} P_{i} X_{i}
\end{aligned}
$$

i.e.

since

$$
V=V_{e}+\delta
$$

$$
\begin{aligned}
\sum_{i=\mathrm{x}}^{n} P_{i} X_{i} & =\sum_{i=1}^{n} x_{i} \sum_{j=1}^{n} a_{i j} P_{j} \\
& =V_{e} \sum_{i=1}^{n} x_{i}=\text { o, by (12). }
\end{aligned}
$$




$$
\begin{aligned}
& V^{\prime}=\sum_{i=\mathbf{x}}^{n} \sum_{j=\mathbf{x}}^{n} a_{i j} p_{i}^{\prime} p_{j}^{\prime} \\
& =\frac{\mathrm{I}}{V^{2}} \sum_{i=\mathrm{I}}^{n} \sum_{j=\mathrm{I}}^{n} a_{i j} p_{i}\left(V_{e}+X_{i}\right) p_{j}\left(V_{e}+X_{j}\right) \\
& =\frac{V_{e}^{2}}{V^{2}} \sum_{i=\mathrm{x}}^{n} \sum_{j=\mathrm{I}}^{n} a_{i j} p_{i} p_{j}+\frac{V_{e}}{V^{2}} \sum_{i=\mathrm{I}}^{n} \sum_{j=\mathrm{I}}^{n} a_{i j} p_{i} p_{j}\left(X_{i}+X_{j}\right)+\frac{\mathrm{I}}{V^{2}} \sum_{i=\mathrm{x}}^{n} \sum_{j=\mathrm{x}}^{n} a_{i j} p_{i} p_{j} X_{i} X_{j} \\
& =\left(\frac{V_{e}}{V}\right)^{2} V+\frac{V_{e}}{V^{2}}\left\{\sum_{i=\mathrm{I}}^{n} p_{i} X_{i} \sum_{j=\mathrm{I}}^{n} a_{i j} p_{j}+\sum_{j=\mathrm{I}}^{n} p_{j} X_{j} \sum_{i=\mathrm{I}}^{n} a_{i j} p_{i}\right\}+\frac{\mathrm{I}}{V^{2}} \sum_{i=\mathrm{I}}^{n} \sum_{j=\mathrm{I}}^{n} a_{i j} p_{i} p_{j} X_{i} X_{j} \\
& =\left(\frac{V_{e}}{V}\right)^{2} V+\frac{2 V_{e}^{2}}{V^{2}} \sum_{i=1}^{n} p_{i} X_{i}+\frac{2 V_{e}}{V^{2}} \sum_{i=\mathrm{I}}^{n} p_{i} X_{i}^{2}+\frac{\mathrm{I}}{V^{2}} \sum_{i=\mathrm{x}}^{n} \sum_{j=\mathrm{I}}^{n} a_{i j} p_{i} p_{j} X_{i} X_{j} \\
& =\left(\frac{V_{e}}{V}\right)^{2}(V+2 \delta)+\frac{2 V_{e}}{V^{2}} \sum_{i=\mathrm{I}}^{n} p_{i} X_{i}^{2}+\frac{\mathrm{I}}{V^{2}} \sum_{i=1}^{n} \sum_{j=\mathrm{I}}^{n} a_{i j} p_{i} p_{j} X_{i} X_{j}
\end{aligned}
$$

Thus

$$
V^{\prime}=V+\frac{2 \delta^{3}-3^{\delta^{2} V}}{V^{2}}+\frac{2 V_{e}}{V^{2}} \sum_{i=\mathrm{I}}^{n} p_{i} X_{i}{ }^{2}+\frac{\mathrm{I}}{V^{2}} \sum_{i=\mathrm{x}}^{n} \sum_{j=\mathrm{I}}^{n} a_{i j} p_{i} p_{j} X_{i} X_{j}
$$

so that

$$
V^{\prime}-V=\frac{2 V_{e}}{V^{2}} \sum_{i=\mathrm{I}}^{n} p_{i} X_{i}^{2}+\frac{\mathrm{I}}{V^{2}} \sum_{i=\mathrm{I}}^{n} \sum_{j=\mathrm{I}}^{n} a_{i j} p_{i} p_{j} X_{i} X_{j}+\mathrm{O}\left(x_{i}^{3}\right)
$$

where $\mathrm{O}\left(x_{i}{ }^{3}\right)$ denotes terms of higher degree than the second in the $x_{i}$.

Consider the terms

$$
\begin{aligned}
& V_{e} \sum_{i=\mathrm{I}}^{n} p_{i} X_{i}^{2}+\sum_{\substack{i, j=\mathrm{I} \\
i \neq j}}^{n} a_{i j} p_{i} p_{j} X_{i} X_{j} \\
& =\sum_{i=\mathrm{I}}^{n} p_{i} X_{i}^{2} \sum_{j=\mathrm{I}}^{n} a_{i j} P_{j}+\sum_{\substack{i, j=\mathrm{I} \\
i \neq j}}^{n} a_{i j} p_{i} p_{j} X_{i} X_{j} \\
& =\sum_{i=\mathrm{I}}^{n} \sum_{j=\mathrm{x}}^{n} a_{i j} P_{i} P_{j} X_{i}^{2}+\sum_{\substack{i, j=\mathrm{I} \\
i \neq j}}^{n} a_{i j} P_{i} P_{j} X_{i} X_{j}+\mathrm{O}\left(x_{i}^{3}\right) \\
& =\sum_{i=\mathrm{x}}^{n} a_{i i} P_{i}^{2} X_{i}^{2}+\sum_{\substack{i, j=\mathrm{I} \\
i<j}}^{n} a_{i j} P_{i} P_{j}\left(X_{i}+X_{j}\right)^{2}+2 \sum_{\substack{i, j=\mathrm{I} \\
i<j}}^{n} a_{i j} P_{i} P_{j} X_{i} X_{j}+\mathrm{O}\left(x_{i}{ }^{3}\right) \\
& =\sum_{i=\mathrm{I}}^{n} a_{i i} P_{i}^{2} X_{i}^{2}+\sum_{\substack{i, j=\mathrm{I} \\
i<j}}^{n} a_{i j} P_{i} P_{j}\left(X_{i}+X_{j}\right)^{2}+\mathrm{O}\left(x_{i}^{3}\right)
\end{aligned}
$$

Thus

$$
\begin{aligned}
\left(V^{\prime}-V\right) V^{2}=V_{e} \sum_{i=\mathrm{I}}^{n} P_{i} X_{i}{ }^{2}+ & 2 \sum_{i=\mathbf{x}}^{n} a_{i i} P_{i}{ }^{2} X_{i}{ }^{2} \\
& +\sum_{\substack{i, j=1 \\
i<j}}^{n} a_{i j} P_{i} P_{j}\left(X_{i}+X_{j}\right)^{2}+\mathrm{O}\left(x_{i}{ }^{3}\right)
\end{aligned}
$$

i.e.

$$
\left(V^{\prime}-V\right) V^{2}=V_{e} \sum_{i=\mathrm{I}}^{n} P_{i} X_{i}^{2}+\frac{1}{2} \sum_{i=\mathrm{I}}^{n} \sum_{j=-\mathrm{I}}^{n} a_{i j} P_{i} P_{j}\left(X_{i}+X_{j}\right)^{2}+\mathrm{O}\left(x_{i}^{3}\right) .
$$


Thus, for all sufficiently small values of the $x_{i}$ (that is, when the population is sufficiently near the equilibrium state) $V^{\prime}-V>0$; this means that the mean viability increases from one generation to the next.

\section{NECESSARY AND SUFFICIENT CONDITIONS FOR STABILITY OF EQUILIBRIUM}

The result obtained in the last section will be used here to obtain sets of necessary and sufficient conditions for a polymorphic equilibrium to be stable.

Consider

$$
V=V\left(p_{1}, p_{2}, \ldots, p_{n}\right)=\sum_{i=1}^{n} \sum_{j=1}^{n} a_{i j} p_{i} p_{j}
$$

as a function of the frequencies $p_{i}$, which are restricted by the condition (2). Introducing the Lagrange undetermined multiplier $\lambda, V$ will be stationary with respect to variations in the $p_{i}$ subject to the restriction (2) whenever

is stationary.

$$
\phi=\phi\left(p_{1}, p_{2}, \ldots, p_{n}\right)=V+\lambda\left\{\sum_{j=1}^{n} p_{j}-\mathrm{I}\right\}
$$

This occurs whenever

which is

$$
\frac{\delta \phi}{\delta p_{i}}=\mathrm{o}, \quad \text { all } i=\mathrm{I}, 2, \ldots, n
$$

$$
2 \sum_{j=1}^{n} a_{i j} p_{j}+\lambda=0 \quad(i=\mathrm{I}, 2, \ldots, n)
$$

But this set is precisely the set of equations (5) which determine the equilibrium gene frequencies $P_{i}$. Hence, if an equilibrium exists, it is a stationary point for $V$.

From this observation and the result obtained in the last section it is easy to see that :

(a) if the equilibrium corresponds to a relative maximum for $V$ then it is stable;

(b) if it corresponds to a minimum value of $V$ then it is unstable ;

(c) if it corresponds to neither a maximum nor a minimum it is either semi-stable or neutral.

In this paper only conditions determining stability will be discussed. These may be obtained by examining the conditions which determine the nature of the stationary value of $V$. Since, by (i6),

$$
V=V_{e}+\delta
$$

it is evident that $V$ has a relative maximum at the equilibrium if and only if the quadratic form

$$
\delta=\sum_{i=1}^{n} \sum_{j=1}^{n} a_{i j} x_{i} x_{j}
$$


is negative definite for all real $x_{i}$ subject to the restriction (I2). A set of necessary and sufficient conditions for this to be the case is (see Ferrar (I95I)):

$$
\begin{aligned}
& \left|\begin{array}{lll}
a_{11} & a_{1 n} & \mathrm{I} \\
a_{n 1} & a_{n n} & \mathrm{I} \\
\mathrm{I} & \mathrm{I} & \mathrm{O}
\end{array}\right|>\mathrm{O}, \quad\left|\begin{array}{llll}
a_{11} & a_{12} & a_{1 n} & \mathrm{I} \\
a_{21} & a_{22} & a_{2 n} & \mathrm{I} \\
a_{n 1} & a_{n 2} & a_{n n} & \mathrm{I} \\
\mathrm{I} & \mathrm{I} & \mathrm{I} & \mathrm{O}
\end{array}\right|<\mathrm{O}, \ldots \ldots \ldots \ldots,
\end{aligned}
$$

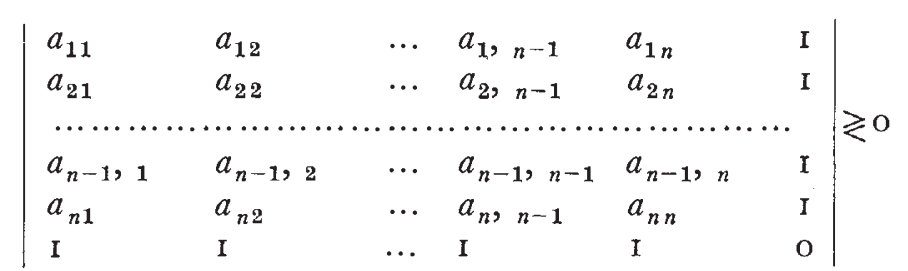

according as $n$ is even or odd respectively.

These conditions, together with the conditions for an equilibrium to exist, viz. :

$$
\text { the } A_{i}(i=\mathrm{I}, 2, \ldots, n) \text { all have the same sign }
$$

are necessary and sufficient for a stable equilibrium.

Introduce the notation

and

$$
D_{r}=\left|\begin{array}{llllll}
a_{11} & a_{12} & \ldots & a_{1 r} & a_{1 n} & \mathrm{I} \\
a_{21} & a_{22} & \ldots & a_{2 r} & a_{2 n} & \mathrm{I} \\
\ldots \ldots & \ldots \ldots \ldots \ldots \ldots \ldots \ldots \ldots \ldots \ldots \ldots \\
a_{r 1} & a_{r 2} & \ldots & a_{r r} & a_{r n} & \mathrm{I} \\
a_{n 1} & a_{n 2} & \ldots & a_{n r} & a_{n n} & \mathrm{I} \\
\mathrm{I} & \mathrm{I} & \ldots & \mathrm{I} & \mathrm{I} & 0
\end{array}\right|
$$

$$
\Delta_{r}=\left|\begin{array}{ccccc}
a_{11} & a_{12} & \ldots & a_{1 r} & a_{1 n} \\
a_{21} & a_{22} & \ldots & a_{2 r} & a_{2 n} \\
\ldots \ldots \ldots \ldots \ldots \ldots \ldots \ldots \ldots \ldots \ldots \ldots \\
a_{r 1} & a_{r 2} & \ldots & a_{r r} & a_{r n} \\
a_{n 1} & a_{n 2} & \ldots & a_{n r} & a_{n n}
\end{array}\right|
$$

Then (25) may be rewritten

$$
(-\mathrm{I})^{r+1} D_{r}>\mathrm{O}, \quad(r=\mathrm{I}, 2, \ldots, n-\mathrm{I})
$$

It will now be shown that an equivalent set of conditions, necessary and sufficient for a stable equilibrium, is (26) together with the sct

$$
(-\mathrm{r})^{r} \Delta_{r}>\mathrm{o}, \quad(r=\mathrm{I}, 2, \ldots, n-\mathrm{I})
$$

For, let

$a_{i j}^{(r)}(i, j=\mathrm{I}, 2, \ldots, r, n)$ denote the cofactor of $a_{i j}$ in $D_{r}(r=\mathrm{I}, 2, \ldots, n-\mathrm{I})$, and $\alpha_{i}^{(r)}(i=\mathrm{I}, 2, \ldots, r, n)$ denote the cofactors of the elements of the last row and column of $D_{r}(r=\mathrm{I}, 2, \ldots, n-\mathrm{I})$. 
Then the determinant which is adjugate to $D_{r}$ has the shape

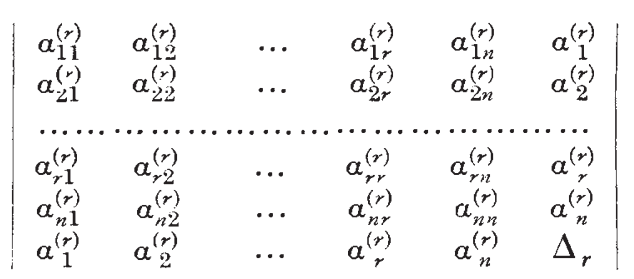

and it is clear that

$$
\alpha_{r r}^{(r)}=D_{r-1} .
$$

Applying Jacobi's Theorem to $D_{r}$ and its adjugate we have

$$
D_{r-1} \Delta_{r}-a_{r}^{()^{2}}=\Delta_{r-1} D_{r}
$$

Beginning with (29), $D_{1}>0$ is simply

whence

$$
2 a_{1 n}>a_{11}+a_{n n}
$$

$$
4 a_{1 n}^{2}>\left(a_{11}+a_{n n}\right)^{2} \geqslant 4 a_{11} a_{n n}
$$

i.e.

$$
\Delta_{1}<0 \text {. }
$$

Then, applying (33) successively for $r=2,3, \ldots, n-\mathrm{I}$, we have $\Delta_{2}>0, \Delta_{3}<0, \ldots,(-1)^{n-1} \Delta_{n-1}>0$, which is (30).

On the other hand, starting with (30), the last equation in this set is

Also

$$
(-\mathrm{I})^{n-1} \Delta_{n-1}>0 \text {. }
$$

$$
D_{n-1}=-\sum_{i=\mathrm{r}}^{n} A_{i} \text {. }
$$

Now

where

$$
\sum_{j=\mathrm{x}}^{n} a_{i j} A_{j k}=\delta_{i k} \Delta_{n-1}, \quad \text { for each } i, k
$$

$$
\left.\begin{array}{rl}
\delta_{i k} & =\mathrm{I} \text { if } i=k \\
& =\mathrm{o} \text { if } i \neq k
\end{array}\right\}
$$

Whence

so that

$$
\sum_{k=\mathrm{x}}^{n} \sum_{j=\mathrm{r}}^{n} a_{i j} A_{j k}=\Delta_{n-1}
$$

that is, using (9)

$$
\sum_{j=\mathrm{x}}^{n} a_{i j} \sum_{k=\mathrm{x}}^{n} A_{j k}=\Delta_{n-1}
$$

$$
\sum_{j=x}^{n} a_{i j} A_{j}=\Delta_{n-1}, \quad \text { for eacn } i .
$$

Since each $a_{i j}$ is positive, at least one of the $A_{j}$ must have the same sign as $\Delta_{n-1}$ and, by condition (26), each $A_{j}(j=\mathrm{I}, 2, \ldots, n)$ has the 
same sign as $\Delta_{n-1}$; finally, by $(36), D_{n-1}$ has the opposite sign to $\Delta_{n-1}$, whence

$$
(-\mathrm{I})^{n} D_{n-1}>0 \text {. }
$$

Successive application of (33) for $r=n-1, n-2, \ldots, 3,2$ yields $(-\mathrm{I})^{n-1} D_{n-2}>0, \ldots, D_{2}<0, D_{1}>0$ which is (29).

Thus (29) and (30), together with the equilibrium conditions (26), are two equivalent sets of conditions, necessary and sufficient for stability. Note that (30) imply (29) subject to (26), whereas the converse is true unconditionally.

In the notation of section 2

$$
V_{e}=\frac{\Delta}{D}=-\frac{\Delta_{n-1}}{D_{n-1}}
$$

since

$$
\Delta_{n-1}=\Delta, \quad D_{n-1}=-D .
$$

From (33) it is at once apparent that

$$
D_{r-1} \Delta_{r} \geqslant \Delta_{r-1} D_{r}, \quad(r=2,3, \ldots, n-\mathrm{I})
$$

so that if the conditions for stability are satisfied

$$
-\frac{\Delta_{r}}{D_{r}} \geqslant-\frac{\Delta_{r-1}}{D_{r-1}} \quad(r=2,3, \ldots, n-\mathrm{I}) . *
$$

Repeated application of $(4 \mathrm{I})$ for $r=n-\mathrm{I}, n-2, \ldots, 3,2$ gives

But

$$
V_{e}>-\frac{\Delta_{1}}{D_{1}}=\frac{a_{1 n}^{2}-a_{11} a_{n n}}{2 a_{1 n}-a_{11}-a_{n n}}
$$

whence

$$
a_{11}^{2}+a_{1 n}^{2} \geqslant 2 a_{11} a_{1 n}
$$

Therefore

$$
a_{1 n}^{2}-a_{11} a_{n n} \geqslant a_{11}\left(2 a_{1 n}-a_{11}-a_{n n}\right)>0 \text {. }
$$

and similarly

$$
V_{e}>a_{11}
$$

$$
V_{e}>a_{i i} \quad(\text { all } i=\mathrm{I}, 2, \ldots, n) .
$$

Thus a necessary condition for a stable equilibrium is that the viabilities of the homozygotes must be less than the mean viability of the population in the equilibrium state. This may be interpreted as a kind of generalised heterosis.

\section{THE SPECIAL CASES OF TWO AND THREE ALLELES}

In this section the case of two alleles, for which the results to be given are well known (see, e.g. Fisher (1922), Haldane (1926) and Wright

* The inequality is strict for $r=n-\mathrm{I}$, since $\alpha_{i}^{(n-\mathrm{r})}=A_{i} \neq 0$ under the conditions for a non-trivial equilibrium; so that $\alpha_{n-1}^{(n-1)^{2}}=A_{n-1}^{2}>0$. 
(193I)), is discussed very briefly; and that of three alleles in some detail.

\section{(i) Two alleles}

Consider two alleles $A, B$ with genotypic viabilities as follows

$\begin{array}{llll}\text { Genotype } & A A & A B & B B \\ \text { Viability } & a & h & b\end{array}$

The viability matrix is

$$
\left(\begin{array}{ll}
a & h \\
h & b
\end{array}\right)
$$

so that, in the notation of section 2 ,

$$
\left.\begin{array}{l}
A_{1}=b-h \\
A_{2}=a-h
\end{array}\right\}
$$

and the condition (26), for an equilibrium to exist, is that these quantities must have the same sign, so that either

$$
\left.\begin{array}{rl}
\text { (i) } & h>a, h>b \\
\text { or (ii) } & h<a, h<b
\end{array}\right\}
$$

The two sets of necessary and sufficient conditions for stability are, in addition to (44), respectively

and

$$
\left|\begin{array}{lll}
a & h & \mathrm{I} \\
h & b & \mathrm{I} \\
\mathrm{I} & \mathrm{I} & \mathrm{o}
\end{array}\right|>\mathrm{O}
$$

of which $(46)$ is

$$
\left|\begin{array}{ll}
a & h \\
h & b
\end{array}\right|<0
$$

$$
(h-a)+(h-b)>0
$$

while (47) is

$$
h^{2}>a b
$$

both of which require (45) (i).

Thus stable equilibrium between two alleles occurs if and only if the heterozygote is more viable than both homozygotes.

\section{(ii) Three alleles}

Consider three alleles $A, B$ and $C$ at a single locus. Using the notation of Owen (1954) the genotypic viabilities are represented by the scheme

\begin{tabular}{l|lll} 
& $A$ & $B$ & $C$ \\
\hline$A$ & $a$ & $h$ & $g$ \\
$B$ & $h$ & $b$ & $f$ \\
$C$ & $g$ & $f$ & $c$
\end{tabular}


Let

$$
\Delta=\left|\begin{array}{lll}
a & h & g \\
h & b & f \\
g & f & c
\end{array}\right|
$$

and let the cofactor of each element in this determinant be represented by the corresponding capitals, so that

$$
A=b c-f^{2}, \quad H=g f-h c, \text { etc. }
$$

Then the condition which must be satisfied for a non-trivial equilibrium to exist is that the three quantities

$$
\left.\begin{array}{l}
A+H+G \\
H+B+F \\
G+F+C
\end{array}\right\}
$$

shall have the same sign.

In addition, the necessary and sufficient conditions for stability are, in the two equivalent forms, respectively

and

$$
\left|\begin{array}{lll}
a & g & \mathrm{I} \\
g & c & \mathrm{I} \\
\mathrm{I} & \mathrm{I} & \mathrm{o}
\end{array}\right|>\mathrm{O},\left|\begin{array}{cccc}
a & h & g & \mathrm{I} \\
h & b & f & \mathrm{I} \\
g & f & c & \mathrm{I} \\
\mathrm{I} & \mathrm{I} & \mathrm{I} & \mathrm{o}
\end{array}\right|<\mathrm{O}
$$

$$
B<\mathrm{o}, \Delta>\mathrm{o} \text {. }
$$

Jacobi's Theorem applied to $\Delta$ and its adjugate reveals that

$$
\left.\begin{array}{rl}
B C-F^{2} & =a \Delta \\
\text { and } A B-H^{2} & =c \Delta
\end{array}\right\}
$$

so that (54) imply also $A<0, C<0$; thus (54) may be stated in the symmetric form

$$
A<\mathrm{o}, B<\mathrm{o}, C<\mathrm{o}, \Delta>\mathrm{o} \text {. }
$$

This is the form in which the conditions are given by Owen (1954).

It is clear, also, that the same argument leads to a corresponding symmetric expression of (53).

Since

$$
\begin{aligned}
\Delta & =(a A+h H+g G)+(a H+h B+g F)+(a G+h F+g C) \\
& =a(A+H+G)+h(H+B+F)+g(G+F+C)
\end{aligned}
$$

it follows that, when $\left(5^{2}\right)$ holds, the condition $\Delta>0$ is equivalent to the condition that any one (and hence all three) of $A+H+G, H+B+F$, $G+F+C$ be positive. Thus yet another set of conditions, necessary and sufficient for a stable equilibrium is $\left(5^{2}\right)$ together with

(i) at least one of $A, B, C<\mathrm{o}$

(ii) at least one of $A+H+G, H+B+F, G+F+C>0$

and if these hold then in each case all three inequalities are true. 
As an example of the application of these conditions, consider the case of three recessive lethals,

i.e. $a=b=c=0$.

For equilibrium it is required that the quantities

$$
f(g+h-f), g(h+f-g) \text { and } h(f+g-h)
$$

should all have the same sign; this requires all three quantities to be positive and $f, g$ and $h$ to satisfy the triangular inequalities

$$
f<g+h, g<h+f, h<f+g .
$$

Moreover (54) are then satisfied automatically so that the conditions (6o) are necessary and sufficient for stability.

In the general case, it is not possible to express the stability conditions in terms of simple inequalities among the viabilities, as in the two-allele problem or the case of lethal homozygotes. The results for two alleles might suggest the analogous relations

$$
a, b, c<f, g, h
$$

(expressing the superiority of each heterozygote over each homozygote) for three alleles; however (6I) is neither necessary nor sufficient for the existence of a stable equilibrium.

For, given $(6 \mathrm{r})$, suppose that

then

$$
a, b, c<f, g<h
$$

$$
G+F+C=(g-a)(f-b)-(h-f)(h-g)<0
$$

for all sufficiently large $h$, so that $(6 \mathrm{I})$ is not sufficient.

On the other hand, the system

$$
\begin{aligned}
& a=\frac{453}{51}, b=0, c=\frac{23}{32} \\
& f=\frac{3}{2}, g=\frac{151}{12}, h=\frac{22}{32}
\end{aligned}
$$

has a stable equilibrium with gene frequencies $\left(\frac{1}{2} \frac{6}{9}, \frac{1}{29}, \frac{1}{2} \frac{2}{9}\right)$ and $h<a, c$; thus $(6 \mathrm{I})$ is also not necessary.

However, the system (6r) does imply the conditions (58), so that if an equilibrium exists under conditions (6I) then it is a stable equilibrium. But, as has been shown above, (6I) is not sufficient to ensure the existence of an equilibrium.

The following conditions which are necessary (but not sufficient) for a stable equilibrium will be established in succession :

(i) No heterozygote is less viable than both the associated homozygotes ; that is, we cannot have $f<b, c$ or $g<c, a$ or $h<a, b$.

(ii) Not more than one of the heterozygotes is less viable than any particular homozygote : that is, one can have, for example

$$
f<a<g, h \text { or } g<a<h, f \text { or } h<a<f, g
$$

but not

$$
f, g<a \text { or } h, f<a \text { or } g, h<a \text {. }
$$


(iii) Furthermore, not more than one heterozygote can be less viable than any of the homozygotes; that is, we cannot have, for example,

$$
c<f<a<g<b<h .
$$

(i) Follows immediately from the necessary conditions $A<0$, $B<$ o, $C<$ o.

(ii) Consider the general condition (43) necessary for a stable equilibrium, i.e.

$$
V_{e}=\frac{\Delta}{D}>a, b, c .
$$

This may be written

$$
\frac{a(A+H+G)+h(H+B+F)+g(G+F+C)}{(A+H+G)+(H+B+F)+(G+F+C)}>a, \text { etc. }
$$

Hence we cannot have

or likewise

or

$$
\left.\begin{array}{l}
g, h<a \\
h, f<b \\
f, g<c
\end{array}\right\}
$$

Suppose now that $h<a, f<a$.

Then, by (66), $g>a$, and from condition (i) $h>b$.

The following possibilities arise :

$$
\begin{aligned}
& \text { (a) } f \geqslant b, f \geqslant c \\
& (\beta) f \leqslant b, f \geqslant c \\
& (\gamma) f \geqslant b, f \leqslant c .
\end{aligned}
$$

If either $(\alpha)$ or $(\beta)$ holds then

$$
H+B+F=(h-a)(f-c)-(g-f)(g-h)<0 .
$$

If $(\gamma)$ holds then by (66) $g>c$ and

$$
\begin{aligned}
H+B+F & =f(g-a)+h(g-c)+a c-g^{2} \\
& <f(g-a)+a(g-c)+a c-g^{2} \\
& =(g-a)(f-g)<0 .
\end{aligned}
$$

Thus in each case the relations $h<a, f<a$ imply $H+B+F<0$ and there is no stable equilibrium; this together with (66) establishes (ii).

(iii) We shall show that each of the inequalities

$$
h<a, h<b, h<c
$$

implics

$$
f, g \geqslant a, b, c \text {. }
$$

For, taking the first case, $h<a$ implies, using (ii), that $f, g>a$.

Suppose now that $f<b$. 
Then, by (ii), $g, h>b$

and this gives rise to the two inequalities

and

$$
\left.\begin{array}{l}
a>h>b \\
b>f>a
\end{array}\right\}
$$

which is impossible. Thus $f \geqslant b$, and the remaining inequalities are established in the same manner.

It follows from (i), (ii), (iii) that there are only four essentially different systems which can give rise to a stable equilibrium. These may be typified as follows :

$$
\left.\begin{array}{l}
\text { (a) } a, b, c<f, g, h \\
\text { (b) } b<h<a, c<f, g \\
\text { (c) } b, c<h<a<f, g \\
\text { (d) } a, b<h<c<f, g
\end{array}\right\}
$$

In $(a)$ all the heterozygotes are more viable than all the homozygotes ; an example of such a system has been given already (see (59), (6o)).

In $(b)$ one heterozygote is less viable than two homozygotes, the non-associated and either of the associated homozygotes. An example is the system $\left(6_{3}\right)$.

In $(c),(d)$ one heterozygote is less viable than just one homozygote; in the former case one of the associated and in the latter the non-associated homozygote. These are exemplified respectively by the following systems :

$$
\begin{aligned}
& a=\frac{5}{8}, b=\mathrm{o}, c=\frac{5}{16} \\
& f=\frac{7}{4}, g=\frac{3}{2}, h=\frac{1}{2}
\end{aligned}
$$

which has a stable equilibrium with gene frequencies $\left(\frac{4}{9}, \frac{1}{9}, \frac{4}{9}\right)$ and $h<a$; and

$$
\begin{aligned}
& a=\frac{5}{16}, b=\mathrm{o}, c=\frac{5}{8} \\
& f=\frac{7}{4}, g=\frac{3}{2}, h=\frac{1}{2}
\end{aligned}
$$

which has a stable equilibrium with gene frequencies $\left(\frac{16}{128}, \frac{59}{128}, \frac{53}{128}\right)$ and $h<c$.

\section{SUMMARY AND CONCLUSIONS}

General conditions have been obtained under which a system of $n$ alleles at a single locus can be maintained in equilibrium by natural selection in a large random mating population. It has been shown that, when the population is in the neighbourhood of such an equilibrium, the mean viability increases from each generation to the next. This establishes a criterion for the stability of the equilibrium, which yields sets of conditions which are necessary and sufficient for stability. In the general case a necessary condition for stability is that the 
viability of each homozygote be less than the mean viability at equilibrium. Roughly speaking, this means that not too many heterozygotes can be less viable than any of the homozygotes. In the twoallele case this is, of course, the classic condition that the heterozygote is fitter than both homozygotes; while in the three-allele case, it has been shown that at most one heterozygous viability may fall below that of at most two homozygotes.

The result concerning the increase in mean viability is in fact true independently of the initial state of the population. This general result depends on an interesting inequality among quadratic forms, which requires a considcrably longer and more difficult proof. The special result proved here is sufficient for the present discussion.

Acknowledgments.--Most of the results concerning the three-allele situation were suggested in unpublished notes by Dr A. R. G. Owen. I wish to thank him for making these available to me and for much helpful advice and encouragement; and the Royal Commission for the Exhibition of 1851 for the award of an Overseas Science Research Scholarship.

\section{REFERENCES}

FERRAR, W. L. I95I. Finite Matrices. Oxford.

Fisher, R. A. I922. On the dominance ratio. Proc. Roy. Soc. Edinb., 42, 32 I-34 I.

HALDANE, J. B. S. 1926. A mathematical theory of natural and artificial selection. Part III. Proc. Camb. Phil. Soc., 23, 363-372.

kimura, m. 1956. Rules for testing stability of a selective polymorphism. Proc. U.S. Nat. Acad. Sci., 42, 336-340.

MANDEL, S. P. H., AND HUGHeS, I. M. I $95^{8}$. Change in mean viability at a multiallelic locus in a population under random mating. Nature, ${ }_{1} 82,63-64$.

owEN, A. R. G. I953. Equilibrium of populations and the possibility of sustained large-scale oscillations. Heredity, 7, I 5 I (abstract).

OWEN, A. R. G. 1954. Balanced polymorphism of a multiple allelic series. Caryologia, Supp. 6, I 240-124I.

PENROSE, L. S., SMITh, S. M., AND SPRotT, D. A. I956. On the stability of allelic systems. Ann. Hum. Genetics, 21, 90-93.

WRIGHT, s. I93I. Evolution in Mendelian populations. Genetics, 16, 97-159. 